\title{
Magnetic Properties of Co-Fe-B Amorphous Films Thermomagnetically Treated with Different Field Directions
}

\author{
Zhe Liu, Weili Li, Weidong Fei, and Dan Xu \\ School of Materials Science and Engineering, Harbin Institute of Technology, Harbin 150001, China \\ Correspondence should be addressed to Weili Li, wlli@hit.edu.cn
}

Received 10 September 2012; Accepted 20 September 2012

Academic Editor: Weichang Hao

Copyright () 2012 Zhe Liu et al. This is an open access article distributed under the Creative Commons Attribution License, which permits unrestricted use, distribution, and reproduction in any medium, provided the original work is properly cited.

Co-Fe-B films were prepared by electroless plating. As-deposited films were thermomagnetically treated in the applied magnetic field of 500 Oe with different field directions at $300^{\circ} \mathrm{C}$ for 1 hour. The effects of magnetic field direction of thermomagnetic treatment on the structure and magnetic properties of Co-Fe-B thin films were investigated. It is found that two phases existed in annealed Co-Fe-B films: one is weak crystallized CoFe phase, the other being amorphous phase. The surface morphologies of the treated films are found to be affected by the direction of thermomagnetic treatment field. The results also show that the magnetic properties of thermomagnetically treated films are influenced greatly by the treatment field direction.

\section{Introduction}

Soft magnetic films have been widely used in magnetic heads, magnetic sensors, and planar inductors at high-frequency range. To play decisive roles in these devices, the magnetic materials must simultaneously possess higher saturation magnetization, lower coercivity, larger magnetic anisotropy, and higher resistivity. Amorphous $\mathrm{Co}-\mathrm{Fe}-\mathrm{B}$ thin films have been found to be good candidates for high-frequency applications, such as magnetic tunnel junction, HDD read-heads, and $\mathrm{GHz}$ magnetic film inductors [1-3]. Varieties of techniques have been used to prepare amorphous $\mathrm{Co}-\mathrm{Fe}-\mathrm{B}$ thin films, such as magnetron sputtering $[4,5]$, electroless deposition [6], and so forth. The electroless deposition method provides an alternative and promising fabrication process with advantages of low-cost, low-energy consumption and adaptation of complicated shapes.

Magnetic field annealing is an efficient way to induce different kinds of anisotropies in some crystalline magnetic materials [7-10], which closely correspond to magnetic performance. It has been reported that the magnetic field annealing can affect the microstructure, domain structure, and magnetic properties of amorphous materials $[8,11]$. The previous researchers $[12,13]$ have indicated that the nanomagnetic phase or cluster can be formed during annealing treatment even when the annealing temperature is below crystallization temperature. In this case, one can expect that the direction of magnetic field may affect the structure and properties of the amorphous films during thermomagnetic treatment. However, there are few reports on the influence of thermomagnetic treatment, especially under small magnetic field with different directions, on the magnetic properties of Co-Fe-B amorphous films.

In this paper, we prepared $\mathrm{Co}-\mathrm{Fe}-\mathrm{B}$ thin films which were annealed in small magnetic field $(500 \mathrm{Oe})$ with respect to the film normal at different angles $\left(\alpha=0 \sim 90^{\circ}\right)$ subsequently. The mechanism of structural relaxation in Co-Fe-B amorphous films induced by thermomagnetic treatment was discussed.

\section{Material and Methods}

Co-Fe-B films were prepared by electroless plating on microscope glass slides $(25 \mathrm{~mm} \times 16 \mathrm{~mm})$ with predeposited $5 \mathrm{~nm}$ thick Ni film as an active layer. The electroless plating solution utilized in this study was the mixture of cobalt sulfate heptahydrate $(0.029 \mathrm{M})$, iron sulfate heptahydrate $(0.009 \mathrm{M})$ as metal sources, sodium borohydride $(0.13 \mathrm{M})$ as reducing agent and boron source, sodium tartrate $(0.239 \mathrm{M})$ as complexing agent, ammonium sulfate $(0.189 \mathrm{M})$, and sodium tetraborate $(0.01 \mathrm{M})$ as additive. During the plating, the solution temperature was maintained at $30 \pm 1^{\circ} \mathrm{C}$ using 


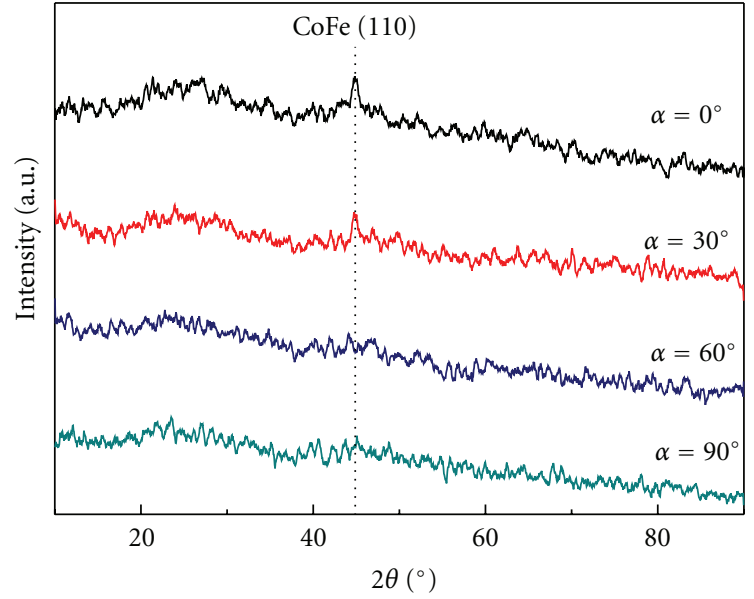

FIGURE 1: GIXRD spectra of the Co-Fe-B thin films thermomagnetically treated with different $\alpha$ angles.

a constant temperature bath and the $\mathrm{pH}$ was maintained constant $(12.7 \pm 0.1)$ without agitation. After 30 min deposition, the sample was removed from the plating solution, then rinsed with distilled water several times and dried with blowing air. The as-deposited film was cut into four pieces $(12.5 \mathrm{~mm} \times 8 \mathrm{~mm})$ in preparation for the following treatment. Structures and magnetic properties of Co-FeB films of varied thickness and composition were studied. Therefore, we focus on the impact of thermomagnetic treatment at different angles on the magnetic properties of Co-Fe-B films with the optimize composition [6] and determined thickness (200 nm).

As-deposited films were then annealed in an applied magnetic field of 500 Oe, oriented differently with respect to the film normal direction at $300^{\circ} \mathrm{C}$ for 1 hour. In order to avoid oxidation during the heat treatment, the films were treated in pure argon gas. The longitudinal direction of all films was kept perpendicular to the field, while the film's normal direction varied from parallel to perpendicular to the field direction during the annealing process. We defined $\alpha\left(0 \sim 90^{\circ}\right)$ as the annealing angle between the film normal direction and the magnetic field direction (corresponds to the films a-d).

Grazing incidence X-ray diffraction (GIXRD) was carried out with the incident angle of $5^{\circ}$ on a Philips X'Pert diffractometer with $\mathrm{Cu} \mathrm{K} \alpha$ radiation generated at $40 \mathrm{kV}$ and $40 \mathrm{~mA}$. Scanning electronic microscopy (SEM) was performed to determine the surface morphology and to estimate the film thickness from cross-sectional views. Vibrating sample magnetometer (VSM) was used to characterize the magnetic properties of the annealed Co-Fe-B films.

\section{Results and Discussion}

As shown in Figure 1, amorphous phase is the main component of all the films after being annealed at different magnetic field directions. When the film normal direction and the magnetic field direction approach to be parallel, that is, $\alpha \leqq 30^{\circ}$, a weak peak can be found around $44.8^{\circ}$, which is

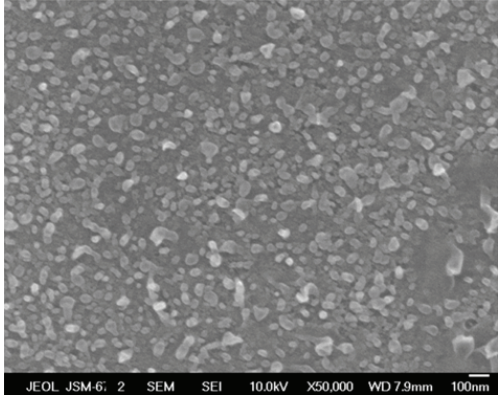

(a)

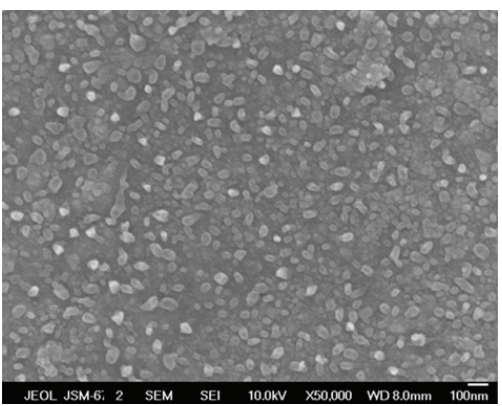

(b)

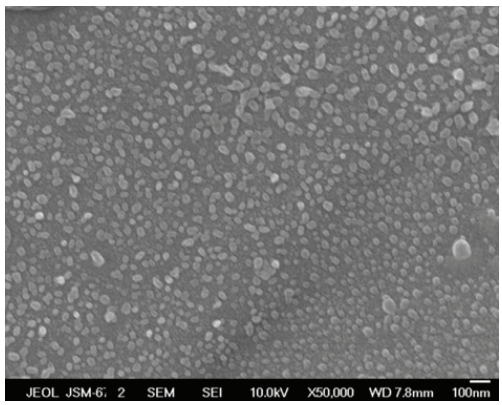

(c)

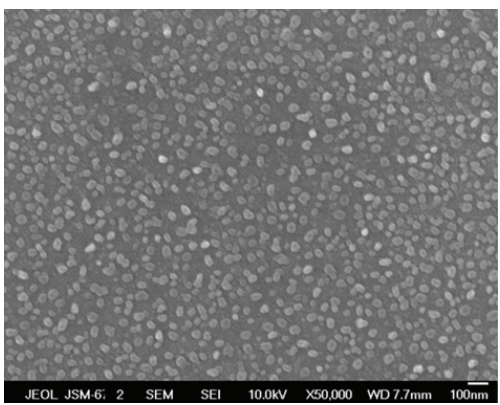

(d)

Figure 2: SEM photos of the Co-Fe-B thin films thermomagnetically treated with different $\alpha$ angles, (a) $\alpha=0^{\circ}$, (b) $\alpha=30^{\circ}$, (c) $\alpha=60^{\circ}$, and (d) $\alpha=90^{\circ}$.

corresponding to CoFe (110) diffraction. The crystallization phase generally has magnetic anisotropy, which is different from amorphous. It is suggested farthing crystalline bcc CoFe phase was formed in amorphous matrix. With the change of magnetic field direction, when $\alpha \geqq 60^{\circ}$, films as-annealed are fully amorphous. 


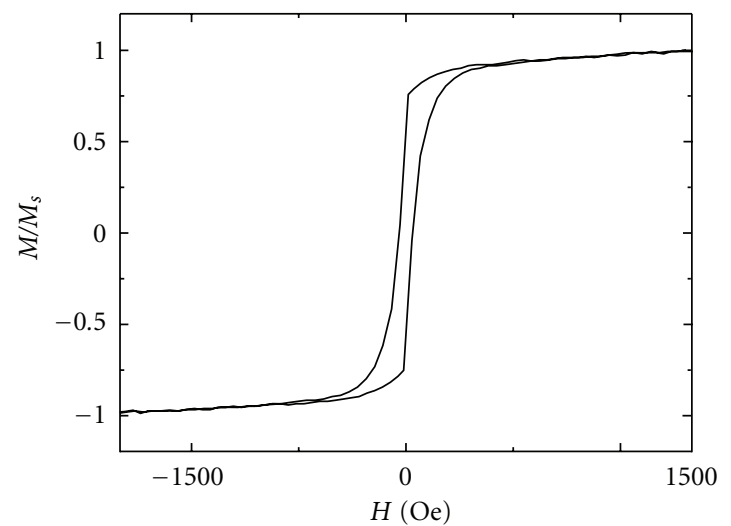

(a)

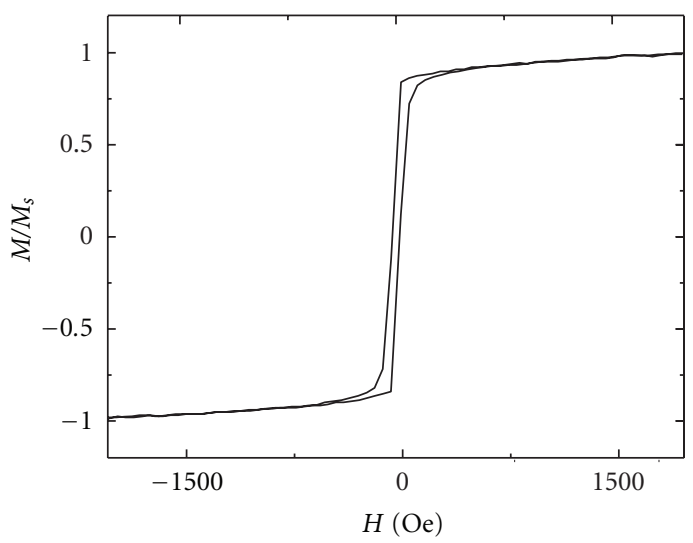

(c)

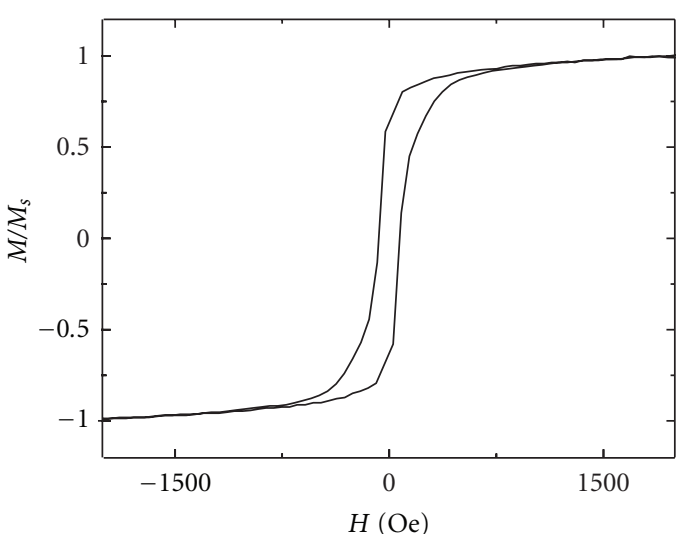

(b)

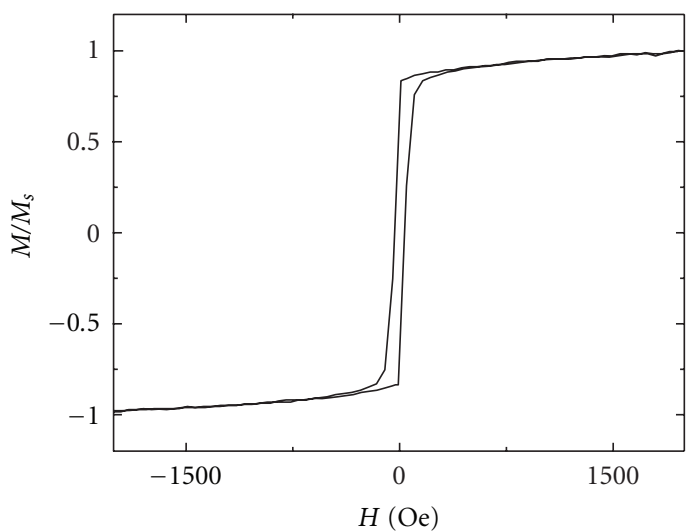

(d)

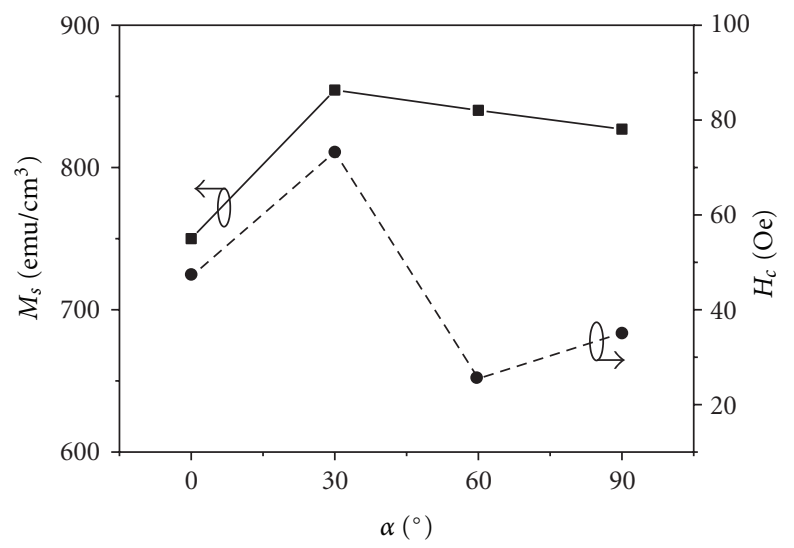

(e)

FIGURE 3: In-plane magnetic hysteresis curves of the Co-Fe-B thin films thermomagnetically treated with different $\alpha$ angles, (a) $\alpha=0^{\circ}$, (b) $\alpha=30^{\circ}$, (c) $\alpha=60^{\circ}$, (d) $\alpha=90^{\circ}$, and (e) the dependences of $M_{s}$ and $H_{c}$ on $\alpha$ angle.

Figure 2 shows the SEM photographs of the Co-Fe-B thin films after thermomagnetic treatment at different angles. It is clear that some tiny particles can be found in all films. The variety of the films' surface morphology after magnetic heat treatment results from local structure relaxation. Under the condition of magnetic field, the short-range order of the amorphous film is enhanced, and the free volume density of the film decreases, resulting in the fluctuation of the surface morphology of the films. For the amorphous films annealed at different magnetic field direction, the conditions of shortrange order and bond structure are different, leading to the differences on the surface morphology of the films treated at different magnetic field directions. Energy dispersion X-ray (EDX) analysis shows that the atomic ratio of $\mathrm{Co}$ and $\mathrm{Fe}$ is $2: 1$.

The in-plane magnetic hysteresis curves of the $\mathrm{Co}-\mathrm{Fe}-\mathrm{B}$ thin films thermomagnetically treated with different $\alpha$ angle are shown in Figure 3. When $\alpha \leqq 30^{\circ}$, the films display 


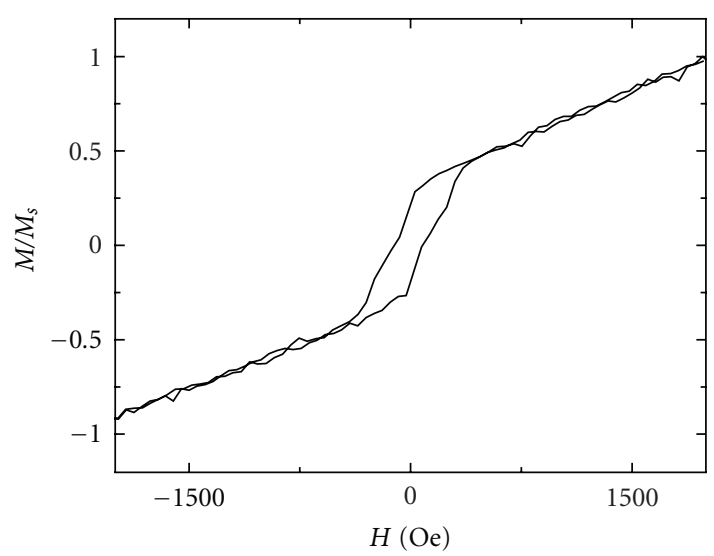

(a)

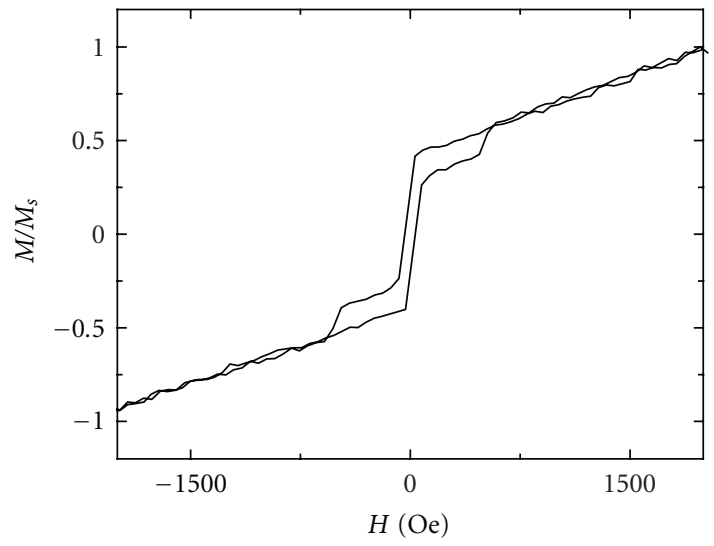

(c)

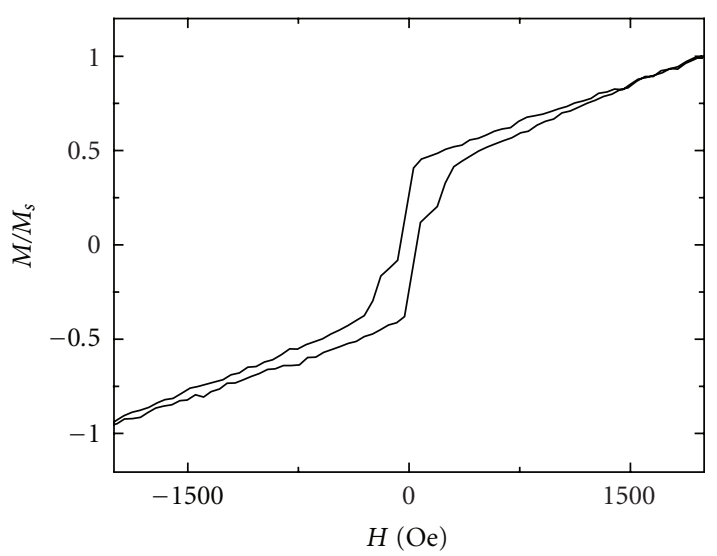

(b)

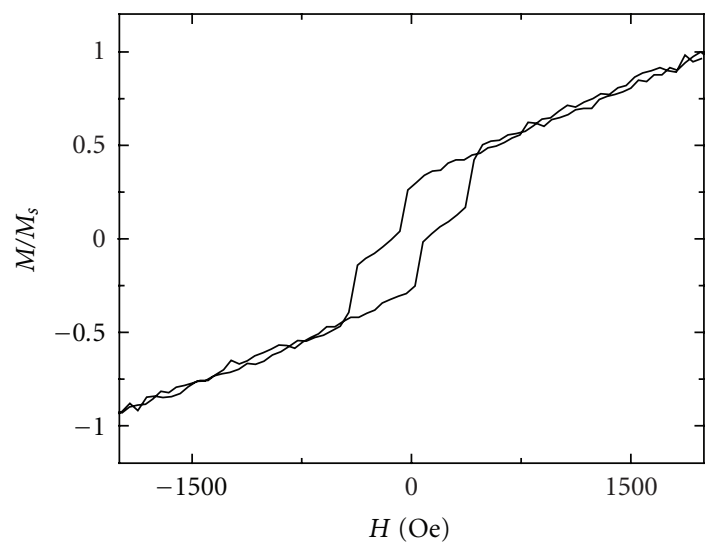

(d)

FIGURE 4: Out-of-plane magnetic hysteresis curves of the Co-Fe-B thin films thermomagnetically treated with different $\alpha$ angles, (a) $\alpha=0^{\circ}$, (b) $\alpha=30^{\circ}$, (c) $\alpha=60^{\circ}$, and (d) $\alpha=90^{\circ}$.

weakly wasp-waisted hysteresis loops. The shapes of loops are approximately square when $\alpha \geqq 60^{\circ}$. Figure 3(e) shows the variation of saturation magnetization and the coercivity as a function of annealing angle measured along the in-plane direction. The maximum of the saturation magnetization and the coercivity reached $854 \mathrm{emu} / \mathrm{cc}$ and 73 Oe when $\alpha=$ $30^{\circ}$, respectively. As the annealing angle increased above $30^{\circ}$, they all descended. Especially, the film annealed at $60^{\circ}$ exhibits lower coercivity and higher saturation magnetization.

As illustrated in Figure 4, the out-of-plane hysteresis loops of the Co-Fe-B thin films thermomagnetically treated with different $\alpha$ angles are very different from those of inplane ones. They also exhibit wasp-waisted shape within the small range of applied magnetic field $( \pm 500 \mathrm{Oe})$. With the annealing angle increases from $0^{\circ}$ to $90^{\circ}$, the wasp-waisted shape becomes more and more obvious.

Thermal annealing of amorphous materials at the temperature well below the crystallization temperature will lead to the change of structure towards a more relaxed state [14]. In the process of structural relaxation, applied magnetic field can affect the anisotropy of the relaxed regions with high magnetization [15]. The easy direction of Fe-Co rich region tends to be parallel to the applied magnetic field to reduce the energy. However, the effective magnetic field in the film is affected by the demagnetization effect in the case of thin film state [16].

In the case of thin films, when $\alpha$ angle is small (i.e., $\alpha \leqq$ $30^{\circ}$ ) for the thermomagnetic treatment, the in-plane applied magnetic field is small, and out-of-plane magnetic field is small as well because of the demagnetization effect. This leads to weak anisotropy in the relaxed regions. However, the effect of in-plane demagnetization field can be neglected when the applied magnetic field is in-plane during thermomagnetic treatment. The in-plane magnetic field applied in the thermomagnetic treatment increases with $\alpha$ angle increasing. The optimum soft magnetic properties obtained in the film thermomagnetically treated with the incline angle of $60^{\circ}$ is the result of the competition between the demagnetization effect and the driving force of annealing magnetic field.

On the basis of the above analysis, it can be concluded that the large magnetic anisotropy induced by thermomagnetic treatment is mainly in-plane when $\alpha$ angle is larger. However, the out-of plane magnetic anisotropy may be weak at small $\alpha$ angle due to the demagnetization effect. When the hysteresis loops were measured in the in-plane direction, the loops are normal ones with square-like shape. Because large 
anisotropy is mainly parallel to the measurement direction. The weak wasp-waisted shape in-plane hysteresis loop at low value of $\alpha$ angle may be caused by the weak anisotropy in the out-of-plane direction [17]. When the hysteresis loops are measured in the out-of-plane direction, the loops for the film thermomagnetically treated with high $\alpha$ angle exhibits more obvious wasp-waisted shape, because of the large magnetic anisotropy in the in-plane direction. The anisotropy constant of Co-Fe-B with an extremely high Curie temperature (about $1300 \mathrm{~K}$ [18]) is big. Therefore, it is hard to form a perpendicular magnetic anisotropy and to get the out-of-plane magnetization saturated (Figures $4(\mathrm{a})-4(\mathrm{~d})$ ). The shape of hysteresis loop merely varied under small magnetic field.

\section{Conclusions}

Co-Fe-B thin films were prepared by electroless deposition successfully. The deposits were annealed in an applied magnetic field of 500 Oe being oriented differently with respect to the direction of film normal at $300^{\circ} \mathrm{C}$ for 1 hour. It is found weak crystallized CoFe phase exist in annealed films. The surface morphologies of annealed films depended strongly on annealing angle.

It is found that magnetic field annealing is an efficient way to obtain different anisotropy in amorphous Co-Fe-B films. In the occasion of small magnetic field annealing, it is found that the optimum soft magnetic properties were not obtained when annealing field was completely parallel to the film plane, but with a little incline $\left(\alpha=60^{\circ}\right)$.

\section{References}

[1] T. Yokoshima, S. Nakamura, D. Kaneko, T. Osaka, S. Takefusa, and A. Tanaka, "Micropattern formation for magnetic recording head using electroless CoFeB deposition," Journal of the Electrochemical Society, vol. 149, no. 8, pp. C375-C382, 2002.

[2] D. D. Djayaprawira, K. Tsunekawa, M. Nagai, H. Maehara, S. Yamagata, N. Watanabe et al., " $230 \%$ room-temperature magnetoresistance in $\mathrm{CoFeB} / \mathrm{MgO} / \mathrm{CoFeB}$ magnetic tunnel junctions," Applied Physics Letters, vol. 86, Article ID 092502, 3 pages, 2005.

[3] M. Munakata, S. I. Aoqui, and M. Yagi, "B-concentration dependence on anisotropy field of $\mathrm{CoFeB}$ thin film for gigahertz frequency use," IEEE Transactions on Magnetics, vol. 41, no. 10, pp. 3262-3264, 2005.

[4] R. D. Gomez, J. S. Ma, A. Arkilic, S. H. Chung, and C. Krafft, "Vortex-antivortex creation and annihilation on CoFeB crosstie patterns," Journal of Applied Physics, vol. 109, no. 7, Article ID 07D310, 2011.

[5] S. A. Manuilov, A. M. Grishin, and M. Munakata, "Ferromagnetic resonance, magnetic susceptibility, and transformation of domain structure in $\mathrm{CoFeB}$ film with growth induced anisotropy," Journal of Applied Physics, vol. 109, no. 8, Article ID 083926, 2011.

[6] C. H. Liu, H. H. He, X. Shen, Y. F. He, and H. H. Li, "Soft magnetic peculiarities of the electroless magnetic loss $\mathrm{CoFeB}$ films," Materials Science and Engineering B, vol. 137, no. 1-3, pp. 103-107, 2007.

[7] S. Chikazumi and T. Oomura, "On the origin of magnetic anisotropy induced by magnetic annealing," Journal of the Physical Society of Japan, vol. 10, no. 10, pp. 842-849, 1955.
[8] B. S. Berry and W. C. Pritchet, "Magnetic annealing and directional ordering of an amorphous ferromagnetic alloy," Physical Review Letters, vol. 34, no. 16, pp. 1022-1025, 1975.

[9] Y. Yoshizawa and K. Yamauchi, "Effects of magnetic field annealing on magnetic properties in ultrafine crystalline FeCu-Nb-Si-B alloys," IEEE Transactions on Magnetics, vol. 25, no. 5, pp. 3324-3326, 1989.

[10] N. Masahashi, M. Matsuo, and K. Watanabe, "Development of preferred orientation in annealing of $\mathrm{Fe}-3.25 \% \mathrm{Si}$ in a high magnetic field," Journal of Materials Research, vol. 13, no. 2, pp. 457-461, 1998.

[11] Y. Yoshizawa, S. Oguma, and K. Yamauchi, "New Fe-based soft magnetic alloys composed of ultrafine grain structure," Journal of Applied Physics, vol. 64, no. 10, pp. 6044-6046, 1988.

[12] S. L. Wang and L. L. Hong, "Effect of the heat treatment on the structure and the properties of the electroless Co-Fe-B alloy," Journal of Alloys and Compounds, vol. 429, no. 1-2, pp. 99-103, 2007.

[13] Y. H. Wang, W. C. Chen, S. Y. Yang et al., "Interfacial and annealing effects on magnetic properties of CoFeB thin films," Journal of Applied Physics, vol. 99, no. 8, Article ID 08M307, 2006.

[14] H. S. Chen, "Glassy metals," Reports on Progress in Physics, vol. 43, no. 4, article 001, pp. 353-432, 1980.

[15] H. Kronmüller, "Magnetic aftereffects, induced anisotropies, and the role of two-level systems in amorphous alloys," Journal of Magnetism and Magnetic Materials, vol. 41, pp. 366-375, 1984.

[16] O. De Abril, M. D. C. Sánchez, and C. Aroca, "The effect of the in-plane demagnetizing field on films with weak perpendicular magnetic anisotropy," Journal of Applied Physics, vol. 100, no. 6, Article ID 063904, 2006.

[17] Q. Gao, G. Hong, J. Ni, W. Wang, J. Tang, and J. He, "Uniaxial anisotropy and novel magnetic behaviors of CoFe2 O4 nanoparticles prepared in a magnetic field," Journal of Applied Physics, vol. 105, no. 7, Article ID 07A516, 2009.

[18] L. Heyne, M. Klaui, D. Backes, P. Mohrke, T. A. Moore, J. G. Kimling et al., "Direct imaging of current-induced domain wall motion in CoFeB structures," Journal of Applied Physics, vol. 103, article 07D928, 2008. 

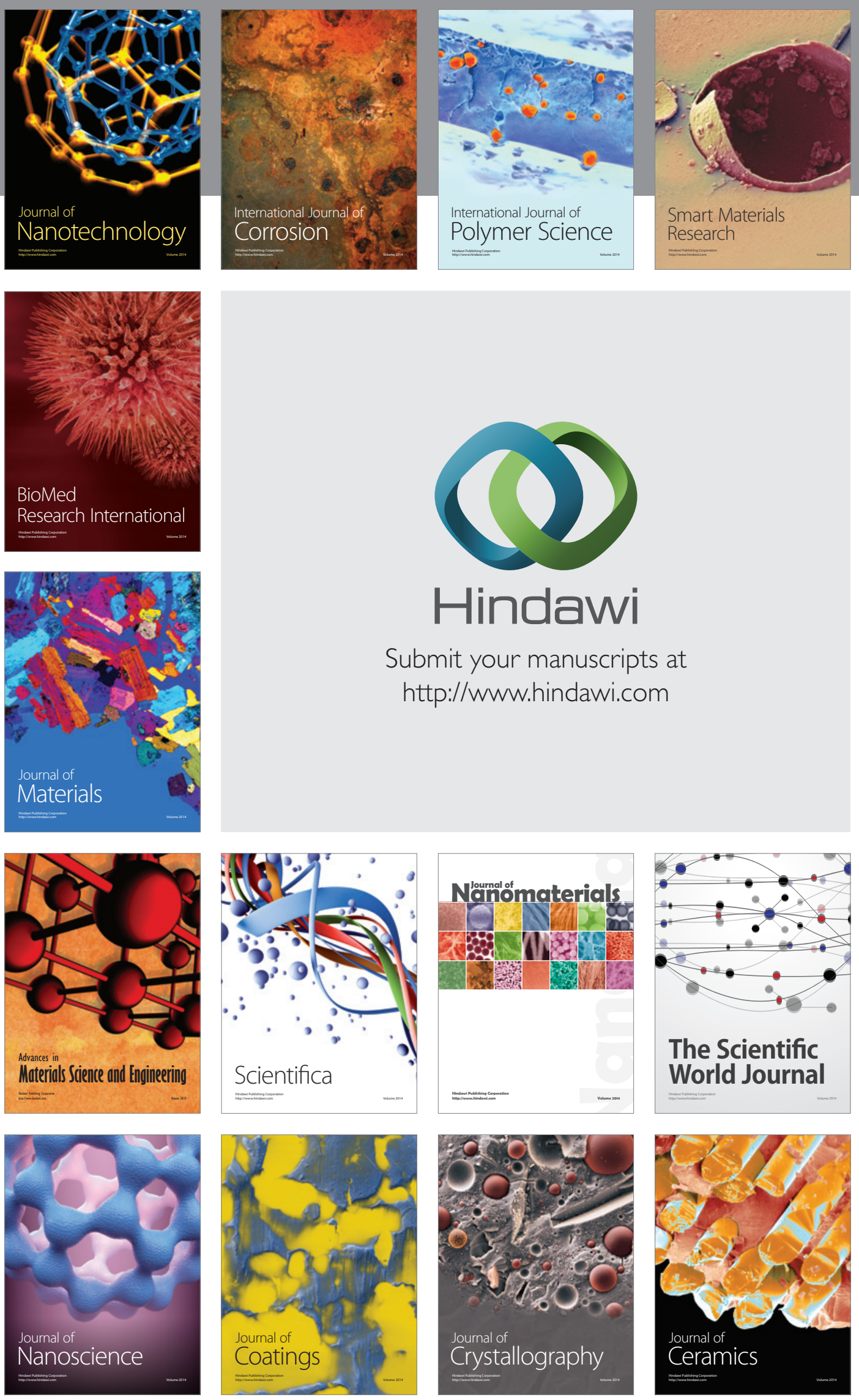

The Scientific World Journal

Submit your manuscripts at

http://www.hindawi.com

\section{World Journal}

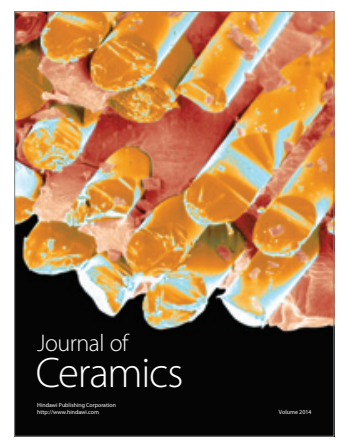

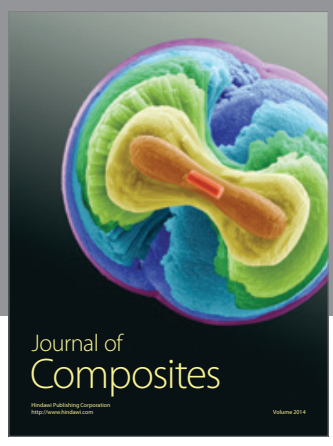
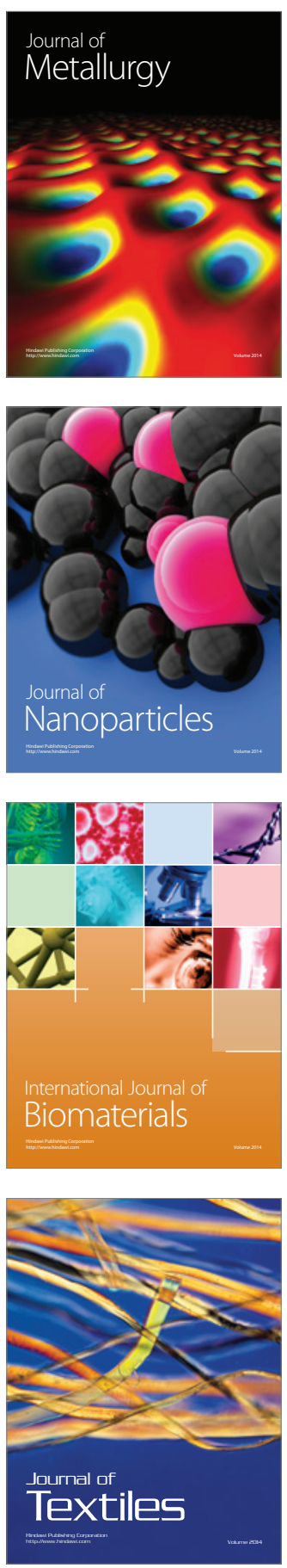